\title{
Análise Explícita de Problemas de Propagação de Ondas Considerando Dissipação Numérica Adaptativa e Subciclagem
}

\author{
Delfim Soares Júnior \\ Danielle Rosâny de Medeiros Leal ${ }^{2}$
}

Faculdade de Engenharia, UFJF, Juiz de Fora, MG

\begin{abstract}
Resumo. A demanda crescente de auxílio computacional em análise de sistemas regidos por Equações Diferenciais representa um grande incentivo ao desenvolvimento de métodos numéricos cada vez mais eficientes. Em particular, problemas de propagação de ondas, considerando análises no domínio do tempo, demandam técnicas avançadas de discretização espacial (e.g., Método dos Elementos Finitos [1] etc.) e temporal (e.g., técnicas explícitas/implícitas de diferenças finitas etc.). Neste contexto, o presente trabalho propõe uma nova técnica explícita de marcha no tempo, contemplando dissipação local e otimizada de modos espúrios, bem como o emprego de discretização temporal local (subciclagem).

Métodos de marcha explícitos são de fácil implementação e exigem baixo custo de processamento e de armazenamento. Também, apesar de serem condicionalmente estáveis, são ideais para a análise de problemas de propagação de ondas, pelo fato de normalmente exigirem um passo de tempo suficientemente pequeno para que tratem adequadamente os modos de alta frequência tipicamente presentes nestes tipos de problemas. Todavia, se por um lado o passo de tempo não pode ser maior que um valor crítico (estabilidade condicionada), este também não deve ser demasiado pequeno, pois o custo computacional está diretamente ligado ao número de passos de tempo que o método utiliza para obter a solução [1]. Então, a utilização de um mesmo passo de tempo para toda a malha de elementos finitos, embora garanta a estabilidade, acaba por subotimizar a marcha no tempo em regiões da malha que não se encontram em condições tão críticas quanto outras. Neste âmbito, a proposta da utilização de múltiplos passos de tempo ao longo da malha, ou subciclagem, surge numa tentativa de melhoria destas questões.

O conceito de subciclagem é recorrente na literatura [4]. Também o é o conceito de métodos explícitos que apresentem dissipação numérica [2, 3]. Ainda, métodos explícitos que possuam dissipação numérica e utilizem subciclagem vêm sendo apresentados recentemente [5, 6]. O presente trabalho propõe a implementação de subciclagem ao novo método proposto por $[7,8]$, sendo este método de fácil implementação, com precisão de segunda ordem, verdadeiramente auto-iniciável, e com controle automático e avançado de dissipação. Assim, a nova proposta se torna de importância, acarretando melhorias ao método, que se torna melhor
\end{abstract}

1delfim.soares@ufjf.edu.br

2danielle.leal@engenharia.ufjf.br 
adaptado a problemas de propagação de ondas, melhorando sua eficiência computacional, sem acarretar perdas em sua generalidade.

$\mathrm{O}$ estudo se encontra em fase inicial e resultados preliminares mostram menor esforço computacional pela diminuição da quantidade e complexidade de cálculos envolvidos na marcha. Resultados esperados são que o novo método tenha maior eficiência computacional e precisão, como já citado, trazendo melhorias à performance da análise.

Palavras-chave. Métodos de Integração no Tempo, Métodos explícitos, Propagação de Ondas, Método dos Elementos Finitos, Dissipação Numérica, Subciclagem

\section{Referências}

[1] K. J. Bathe, Finite Element Procedures, Prentice-Hall, Upper Saddle River, New Jersey, (1996).

[2] J. Chung and G. M. Hulbert, A time integration algorithm for structural dynamic improved numerical dissipation: the generalized- $\alpha$ method, J. Applied Mechanics, vol. $60,371-375$, (1993).

[3] J. Chung and J. M. Lee, A new family of explicit time integration methods for linear and non-linear structural dynamics, International J. for Numerical Methods in Engineering, vol. 37, 3961-3976, (1994).

[4] M. A. Dokainish and K. Subbaraj, A survey of direct time-integration methods in computational structural dynamics - I. explicit methods, Computer \& Structures, vol. 32, No. 6, 1371-1386, (1989).

[5] M. J. Grote and T. Mitkova, Explicit local time-stepping methods for Maxwell's equations, J. of Computational and Applied Mathematics, vol. 234, 3283-3302, (2010).

[6] L. Mey and Y. Chen, Explicit multistep method for the numerical solution of RLW equation, Applied Mathematics and Computation, vol. 218, 9547-9554, (2012).

[7] D. Soares, An explicit family of time marching procedures with adaptive dissipation control. International Journal for Numerical Methods in Engineering, vol. 100, 165-182, (2014).

[8] D. Soares, An explicit time marching technique with solution-adaptive time integration parameters, Technical Report, UFJF, Juiz de Fora, (2014). 